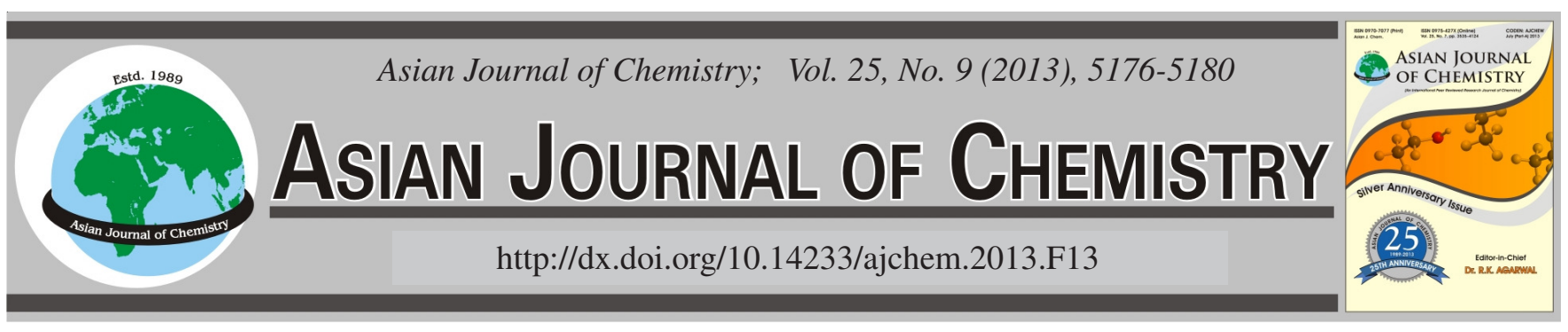

\title{
Effect of the Particle Orientation on the Magneto-Rheological Behaviour of Carbonyl Iron Powder-Filled Natural Rubber $\dagger$
}

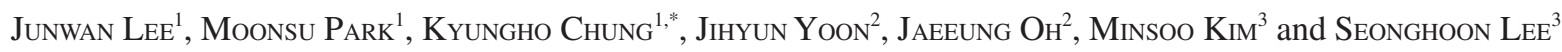

\author{
${ }^{1}$ Department of Polymer Engineering, The University of Suwon, Suwon, Republic of Korea \\ ${ }^{2}$ School of Mechanical Engineering, Hanyang University, Seoul, Republic of Korea \\ ${ }^{3}$ Hyundai Mortor Co., Hwaseong, Republic of Korea \\ *Corresponding author: Tel: +82 312202109 ; E-mail: khchung@ suwon.ac.kr
}

AJC-13167

\begin{abstract}
Compared to a conventional rubber vibration isolator, a magneto-rheological elastomer (MRE) can absorb a broader frequency range of vibrations. The characteristic phenomena results from the orientation of magneto responsible particles via chain-like formation in a rubber matrix. In this study, natural rubber was used as the matrix to manufacture magneto-rheological elastomers. Carbonyl iron powder, which has a rapid magnetic reaction, was selected as the magnetic particle to create a magnetic field reactive modulus. The mechanical properties of the manufactured magneto-rheological elastomers were measured without the application of a magnetic field. With the addition of carbonyl iron powder, the tensile property decreased and the hardness increased. The magneto-rheological effect was analyzed using a fast Fourier transform analyzer with various magnetic fluxes. The magneto-rheological effect also increased with increasing carbonyl iron powder content and magnetic flux. In addition, the carbonyl iron powder loading affected the mechanical properties of magneto-rheological elastomer. A modulus variable vibration isolator can be developed if the mechanical properties and manufacturing process of magneto-rheological elastomer are controlled properly by the magnetic field.
\end{abstract}

Key Words: Magneto-rheological elastomer, Rubber vibration isolator, Carbonyl iron powder, Fast Fourier transform analyzer.

ᄂ _ - _ - - - - - - - - - - - - - - - - - - - - - - -

\section{INTRODUCTION}

From automotives to electronic devices, rubber vibration isolators (RVIs) are used with multi-utilities and play important roles. On the other hand, the efficient control of variably generated vibrations is difficult because these conventional rubber vibration isolators can only absorb a restricted range of frequencies due to their inherent modulus. Therefore, a number of studies on the efficient control of variably generated vibrations have been performed with the magneto-rheological elastomer (MRE) being one solutions ${ }^{1-3}$.

Magneto-rheological elastomer is a composite in which the rubber matrix is filled with magneto responsible particles (MRP) so that the modulus of the composite can be varied according to applied the magnetic field. These unique phenomena can help reveal the chain like formation of filled magneto responsible particle along with the direction of the applied magnetic field ${ }^{4,5}$. If a magnetic field is applied to a composite before curing the matrix, magneto responsible particles dispersed randomly in the matrix can be arranged along the direction of the magnetic field. This results in a broader absorbable frequency range. The magneto-rheological fluid (MRF), whose matrix is viscous fluid, is another modulus variable material with a similar mechanism. On the other hand, the relatively lower modulus of the fluid matrix, the settlement of minute magneto responsible particle and the need for a fluid container can be problematic ${ }^{6-9}$. These problems can be solved by replacing the viscous fluid matrix with an elastic solid matrix. The matrix material, magneto responsible particle concentration and magnetic flux density are factors that affect the change in the modulus of MRE ${ }^{10,11}$.

In this study, natural rubber was used as an elastomeric matrix and micro sized magneto responsible particle at various concentrations was used as the filler to study the magnetorheological effect and mechanical properties of MRE. The magneto-rheological properties of MRE were examined by applying a range of external magnetic fields on MREs.

\section{EXPERIMENTAL}

Materials and preparation of MRE specimen: NR (natural rubber, RSS\#1) was used as the matrix of MRE. The carbonyl iron powder, which was used to induce the magneto- 
rheological property, were spherical particles with a mean diameter of 3-5 $\mu \mathrm{m}$. Table-1 shows the composition of MRE. Compounding was processed with an open-roll mill at room temperature. After the mastication of natural rubber, $\mathrm{ZnO}$ and stearic acid were added followed by the addition of sulfur and an accele-rator. The final compound of MRE was prepared by the addition of carbonyl iron powder and stored at room temperature for $24 \mathrm{~h}$ for stabilization. The cure characteristics of the compound were measured using a rubber rheometer (DAEKYUNG, DRM-100, Korea). Vulcanization was carried out using a hydraulic hot press. The tensile specimen was cut into a dumbbell shape. The length, width and thickness of the transmissibility measurement (estimation of damping property of MRE) specimen were $20 \mathrm{~mm} \times 20 \mathrm{~mm} \times 2 \mathrm{~mm}$ of, respectively.

\begin{tabular}{cc} 
TABLE-1 \\
COMPOSITION OF THE MRE MATRIX \\
\hline Ingredients & Amounts (phr) \\
\hline Natural rubber & 100 \\
ZnO & 5 \\
Stearic acid & 2 \\
N-cyclohexyl-2-benzothiazolesulfenamide & 0.8 \\
Sulfur & 2.5 \\
\hline
\end{tabular}

Three types of MRE were prepared for comparison. The isotropic MRE (i-MRE) was prepared by the homogeneous distribution of carbonyl iron powder on the matrix. The anisotropic MREs (a-MRE1 and a-MRE2) were prepared by two methods. A-MRE1 was prepared using a neodymium magnet for the carbonyl iron powder orientation. The MRE compound sheet with a $2 \mathrm{~mm}$ thickness was prepared using a hydraulic press. The sheet was placed between two Nd magnets. The surface magnet density was approximately 0.6 Tesla. The sheet was pretreated at $80^{\circ} \mathrm{C}$ for $1 \mathrm{~h}$. MRE was cured at $160{ }^{\circ} \mathrm{C}$ for $0.5 \mathrm{~h}$. The other case used the newly designed mold and the material was designated as a-MRE2. The details of a-MRE2 will be reported in a later section. The orientation of carbonyl iron powder with the application of an external magnetic field was examined by scanning electron microscopy (SEM, JSM5200, JEOL).

Mechanical properties of MRE: The tensile properties of MRE were examined at room temperature in the absence of an external magnetic field. The strain rate was $2.5 \mathrm{~min}^{-1}$. The hardness of MRE with the variation of composition was measured using a Shore A durometer.

Damping property of MRE: The damping property of MRE with the amounts of carbonyl iron powder was examined using a self-modified Fast Fourier Transform (FFT) analyzer. Fig. 1 shows the structure of the FFT analyzer. The electromagnet applied FFT analyzer was equipped with a fixed end beam for stable measurement. The lower accelerator measured the vibration generated from the base. The upper accelerator measured the transferred vibration through the modulus variable MRE using the magnetic flux generator. The oscillator gave a shear force on MRE as a load by inertia. White noise excitation applied to the entire system was generated using a shaker beneath the fixed-fixed end beam to examine the shear modulus of MRE due to the induced current. When the entire system was excited, MRE connecting the magnetic flux generator and oscillator deformed in the shear direction. The natural frequency shift of oscillator occurred as a result of changing the shear stiffness of MRE. The measured signals were sent to the FFT analyzer. By this process, the transmissibility change in MRE with the amounts of carbonyl iron powder was measured under a constant magnetic field. In addition, the change in transmissibility with the various input currents was measured on MRE containing 30 vol. \% carbonyl iron powder.

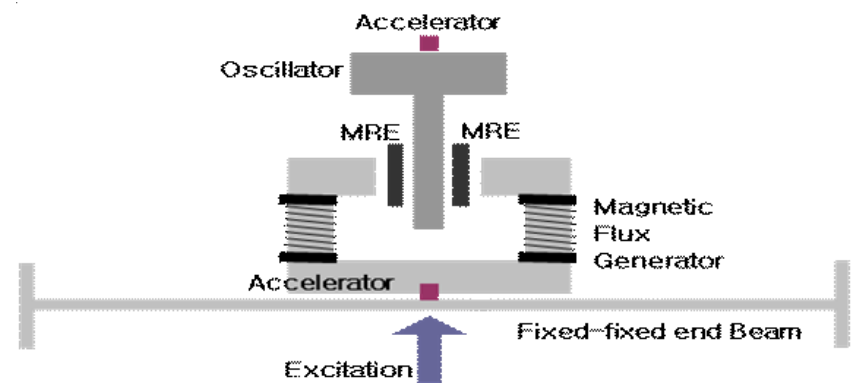

Fig. 1. Self-modified electromagnet applied fast Fourier transform analyzer

\section{RESULTS AND DISCUSSION}

Morphology of MRE: The difference in morphology between $\mathrm{i}-\mathrm{MRE}$ and a-MRE suggests that $\mathrm{i}-\mathrm{MRE}$ has a homogeneous distribution of carbonyl iron powder, whereas a-MRE showed the chain-like formation of carbonyl iron powder along the magnetic field. Although the manufacturing process of a-MRE1 was a convenient process using a Nd magnet, several problems still remain. Separation of the Nd magnet was quite difficult after inducing the orientation of the carbonyl iron powder. The rearrangement of the carbonyl iron powder orientation occurred when the MRE sheet was cured under press. In addition, many voids were observed inside of a-MRE1, as shown in Fig. 2. Fig. 2a and 2b show cross-sections of a-MRE1 and i-MRE, respectively. a-MRE1 contained many voids. In the case of a-MRE1, the bumping action during the curing process was restricted to minimize the rearrangement of the pre-oriented carbonyl iron powder. Therefore, another process for manufacturing a-MRE was proposed to solve the inherent problems of a-MRE1. The mold inserted $\mathrm{Nd}$ magnet was proposed for curing the a-MRE in this study. Fig. 3 shows the design diagram and a photograph of the mold. The a-MRE manufactured by this method was designated a-MRE2. The dimensions of the $\mathrm{Nd}$ magnet were $600 \mathrm{~mm} \times 120 \mathrm{~mm} \times 350$ $\mathrm{mm}$ and the $\mathrm{Nd}$ magnet was coated with a $3 \mathrm{~mm}$ thick aluminum layer to protect the magnet. The surface magnetic density of the $\mathrm{Nd}$ magnet was 0.2-0.3 Tesla.

The superior magneto-rheological effect of a-MRE might be due to the chain-like formation of carbonyl iron powder on the matrix by the magnetic field. Fig. 4 shows SEM images of various MREs. i-MRE containing 30 vol. \% carbonyl iron powder had a homogeneous distribution of carbonyl iron powder in the matrix (Fig. 4a). The morphology of a-MRE1 (Fig. 4b) was similar to that of i-MRE. Although the Nd magnet was used to induce the orientation of carbonyl iron powder on the matrix, the orientation of carbonyl iron powder disappeared due to a bumping process during curing of MRE. The bumping 


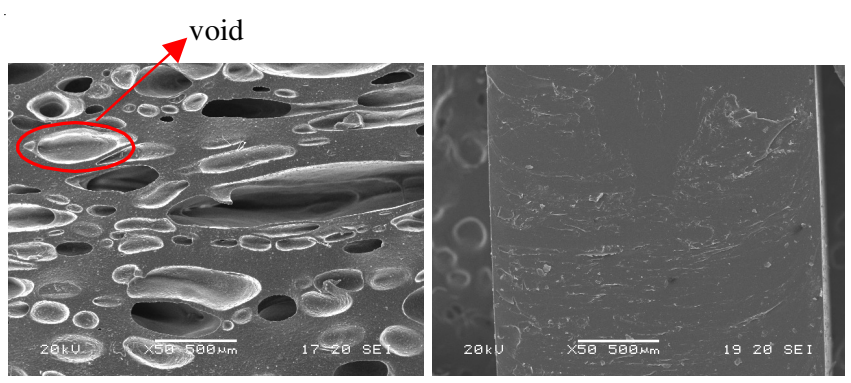

Fig. 2. Cross-section of MRE: (a) a-MRE1, (b) i-MRE
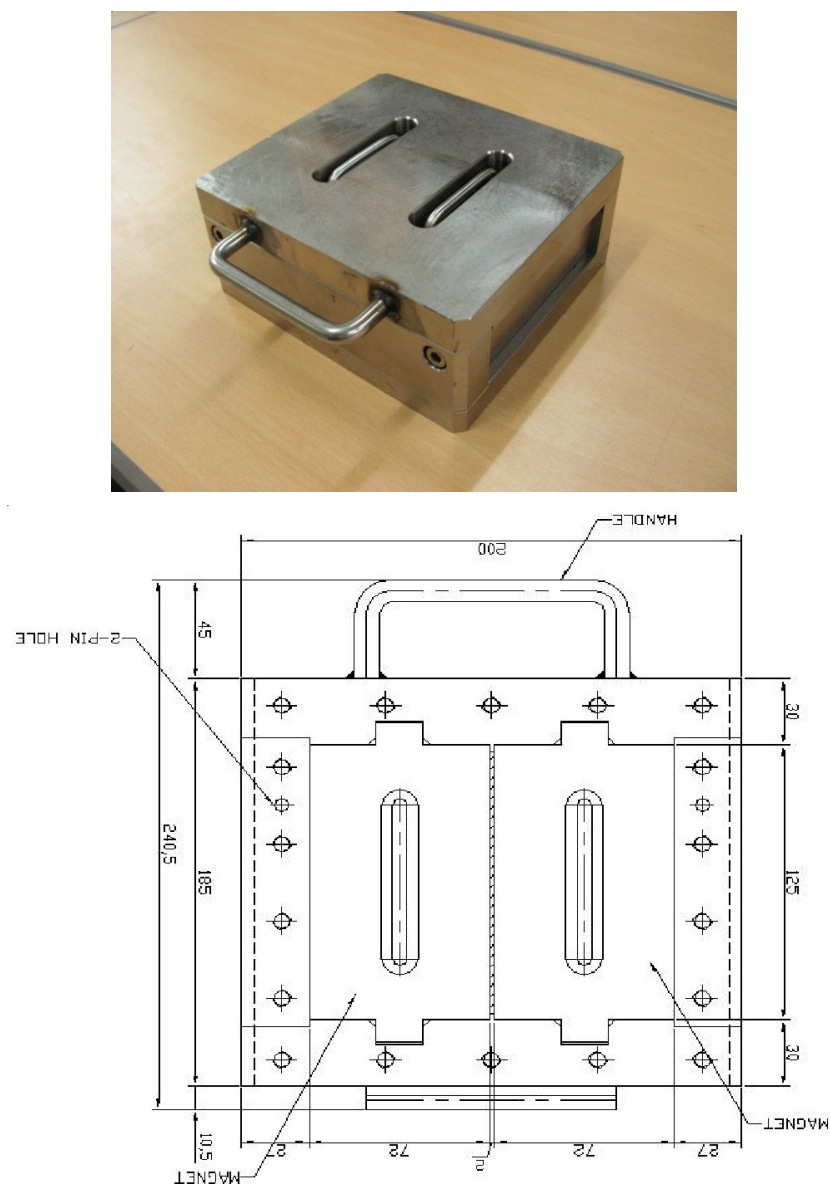

Fig. 3. Design of the neodymium magnet inserted mold

process was performed to reduce the possibility of void occurrence on MRE matrix. On the other hand, the apparent orientation of carbonyl iron powder was observed in a-MRE2, as shown in Fig. 4c. In the case of a-MRE2, the carbonyl iron powder orientation was maintained during the curing process of MRE, even though the bumping process was applied to prevent the generation of voids. This was attributed to the continuous application of a magnetic field during the bumping process. Therefore, it is expected that a-MRE2 would show the best magneto-rheological effect.

Mechanical properties of MRE: The tensile property of i-MRE with the loading amounts of carbonyl iron powder was examined. The amounts of carbonyl iron powder ranged from 0 to 50 vol. \%. The results are shown in Fig. 5. The tensile strength and elongation increased with the addition of carbonyl iron powder due to the reinforcing effect of the spherical carbonyl iron powder particles (Fig. 5a). With increasing carbonyl iron powder loading, MRE exhibited typical tensile failure envelop behaviour. The tensile properties of MRE was superior to the basic rubber compound without carbonyl iron powder until $30 \mathrm{wt}$. \%. In terms of the MR effect, a better MR effect was expected with increasing carbonyl iron powder loading. On the other hand, a decrease in the mechanical properties with increasing carbonyl iron powder loading above the optimum amounts should be considered. Therefore, the optimum carbonyl iron powder content in MRE might be $30 \mathrm{wt}$ \% because the MR effect would increase with increasing carbonyl iron powder content. Fig. 5b shows very interesting tensile results of a-MRE2. In contrast to the results of i-MRE, the tensile strength of a-MRE2 decreased with increasing carbonyl iron powder content. This was attributed to the orientation of carbonyl iron powder. The orientational direction of carbonyl iron powder on a-MRE specimen was perpendicular to the direction of the tensile direction. Therefore, it is believed that the lower tensile strength of a-MRE would result in a more orientated carbonyl iron powder in a-MRE. A comparison of the data in Fig. 5 showed that the tensile strength of a-MRE was lower than that of i-MRE and a different trend with the carbonyl iron powder loading was observed.

Table-2 lists the change in MRE hardness with the addition of carbonyl iron powder. The hardness of MRE increased linearly with increasing carbonyl iron powder content regardless of the type of MRE. The hardness of a-MRE was higher than that of i-MRE when both MREs containing the same carbonyl iron powder loading were compared. The reason why a-MRE showed higher hardness than i-MRE was the measuring direction of the hardness. The hardness was measured along the orientation direction of carbonyl iron powder. Along with the results of the tensile strength, the hardness of MRE is an

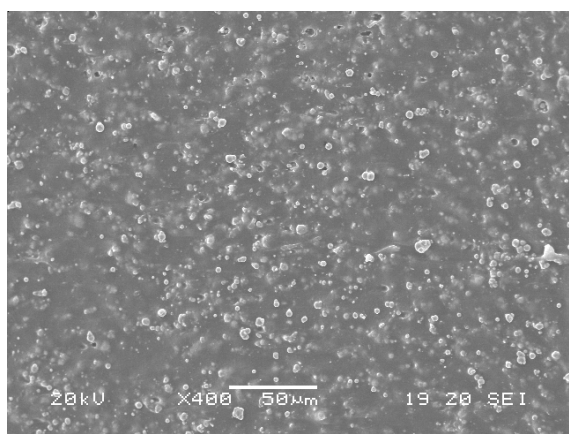

(a)

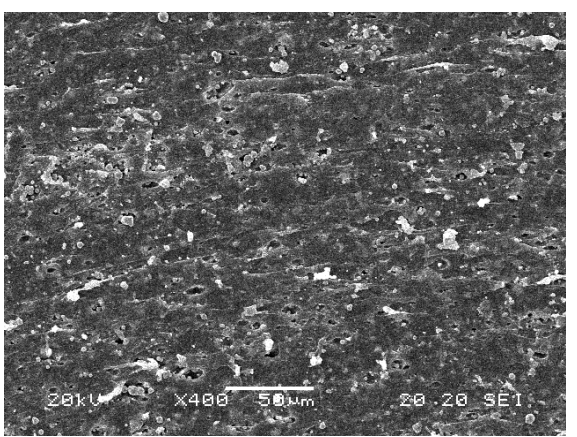

(b)

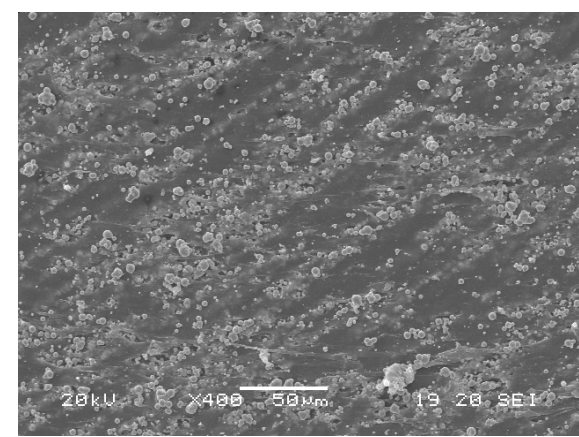

(c)

Fig. 4. Morphology of the MREs : (a) i-MRE; (b) a-MRE1; (c) a-MRE2 


\begin{tabular}{ccc}
\hline & \multicolumn{2}{c}{ TABLE-2 } \\
HARDNESS OF MRE CONTAINING VARIOUS \\
AMOUNTS OF CARBONYL IRON POWDER (CIP)
\end{tabular}

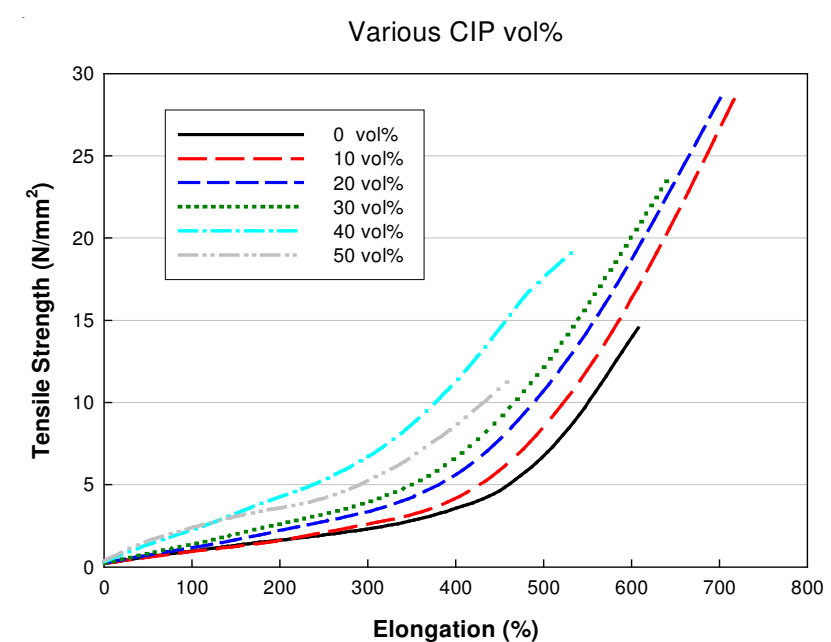

(a)

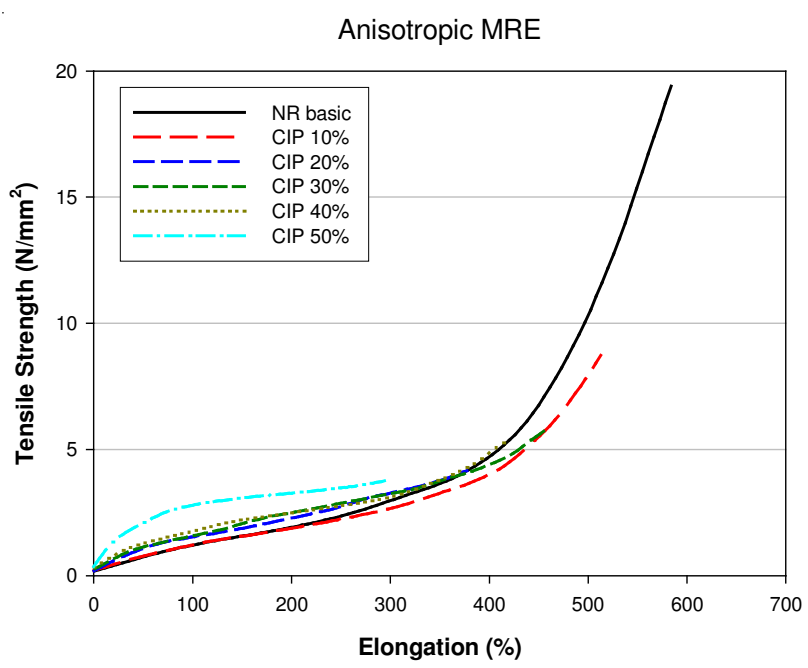

(b)

Fig. 5. Tensile property of MRE with the amounts of carbonyl iron powder: (a) i-MRE; (b) a-MRE

important factor for estimating the orientation of carbonyl iron powder.

Magneto-rheological effect of a-MRE: The damping effect of fabricated MRE was measured with various carbonyl iron powder concentrations and various external magnetic fields. Fig. 6 shows the change in transmissibility of MRE with various carbonyl iron powder loadings at a magnetic field of 0.078 T. Fig. 7 presents the transmissibility change in 30 vol \% carbonyl iron powder filled MRE with various external magnetic field densities. Table-3 lists the minimum values of the transmissibility with the carbonyl iron powder content and

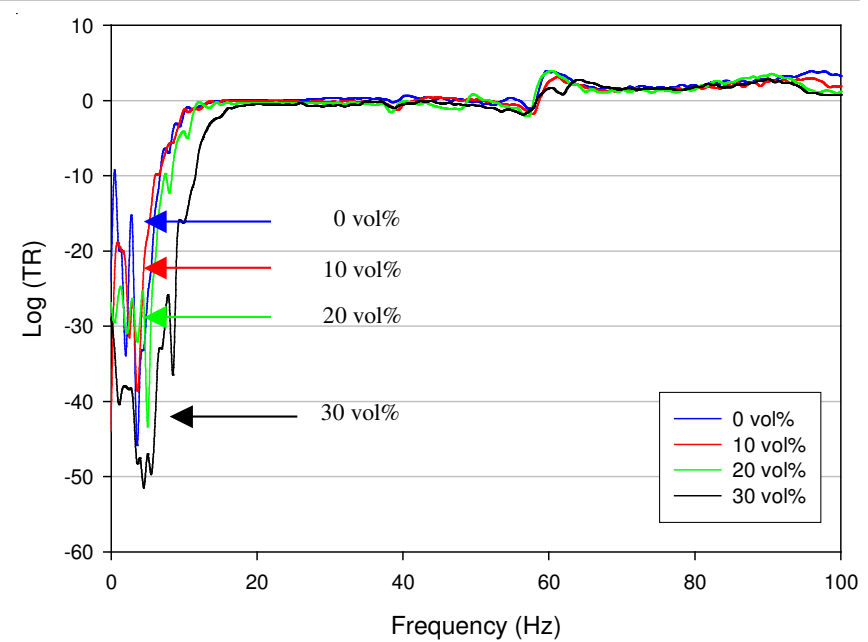

Fig. 6. Transmissibility changes of MRE with the amounts of carbonyl iron powder under $0.078 \mathrm{~T}$ magnetic field

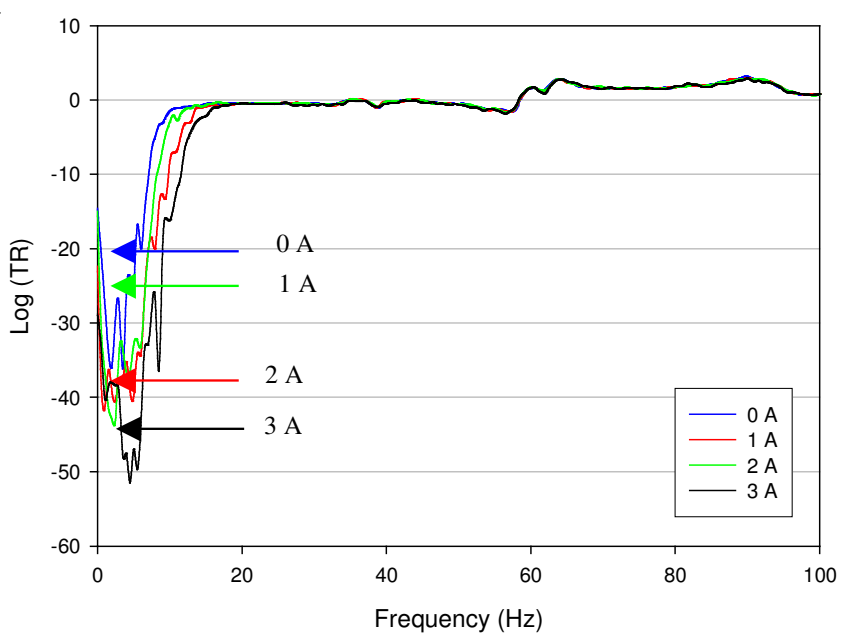

Fig. 7. Transmissibility changes of 30vol\% carbonyl iron powder filled MRE with various external magnetic fields

\begin{tabular}{ccc} 
TABLE-3 & \\
MINUMUM TRANSMISSIBILITY OF MRE WITH VARIOUS \\
CIP CONTENTS AND MAGNETIC FIELD DENSITIES \\
\hline & 0 & $\log (\mathrm{TR})$ \\
\hline & 10 & -45.33 \\
CIP (vol.\%) & 20 & -43.89 \\
& 30 & -43.39 \\
& 0 & -51.51 \\
\hline Input current & 1 & -36.17 \\
(amphere) & 2 & -41.39 \\
& 3 & -43.01 \\
\hline
\end{tabular}

TABLE-4

MAGNETIC FIELD DENSITY WITH THE INPUT CURRENT

$\begin{array}{lllll}\text { Input current (Amphere) } & 0 \mathrm{~A} & 1 \mathrm{~A} & 2 \mathrm{~A} & 3 \mathrm{~A}\end{array}$

\begin{tabular}{lllll}
\hline Magnetic field density (Tesla) & $0 \mathrm{~T}$ & $0.033 \mathrm{~T}$ & $0.056 \mathrm{~T}$ & $0.078 \mathrm{~T}$
\end{tabular}

magnetic field densities. Table-4 presents the external magnetic fields with the input currents. The transmissibility is the ratio of the generated vibration to the transferred vibration. Therefore, a smaller log (TR) indicates a better damping effect. Fig. 7 shows that the transmissibility decreased with increased magnetic field density. The change in the transmissibility and 
vibration absorption range resulted from the chain-like formation of carbonyl iron powder, which was filled in the natural rubber matrix. Therefore, the carbonyl iron powder concentration and external magnetic field are major factors that can control the damping property of MRE.

\section{Conclusion}

The tensile properties and hardness of MRE were measured. A more oriented carbonyl iron powder in MRE matrix resulted in lower tensile strength and higher hardness. Therefore, the orientation of carbonyl iron powder was confirmed indirectly by measuring the mechanical properties, such as the tensile strength and hardness. The damping property of MRE with various external magnetic fields and carbonyl iron powder concentrations were measured using a FFT analyzer. The maximum transmissibility of MRE was observed at the external magnetic field of $0.078 \mathrm{~T}$ and with the addition of 30 vol \% carbonyl iron powder. The chain-like formation of carbonyl iron powder in the natural rubber matrix is a key mechanism in controlling the damping property of MRE.

\section{ACKNOWLEDGEMENTS}

This study was supported by the Basic Science Research Program through the National Research Foundation of Korea (NRF) funded by the Ministry of Education, Science and Technology (MEST, D00256). The authors appreciated the partial support from Hyundai Kia Motor Company.

\section{REFERENCES}

1. J.D. Carlson and M.R. Jolly, Mechatronics, 10, 555 (2000).

2. S.J. Park and K.E. Choi, Elastom. Compos., 46, 10 (2011).

3. G.Y. Kim and S.C. Park, Elastom. Compos., 45, 291 (2010).

4. M. Jolly, J. Carlson and B. Munoz, Smart Mater. Struct, 5, 607 (1996).

5. K.H. Chung and K.S. Yoon, Elastom. Compos., 45, 106 (2010).

6. T. Singa, A. Okada and T. Kurauchi, J. Appl. Polym. Sci., 58, 787 (1995).

7. M. Jolly, J. Carlson, B. Munoz and T. Bullions, J. Int. Mat. Syst. Struct., 7, 613 (1996).

8. J. Ginder, M. Nichols, L. Elie and J. Tardiff, SPIE, 3675, 131 (1999).

9. M. Nichols, J. Ginder, J. Tardiff and L. Elie, In Proceedings of 156th ACS Rubber Division Meeting, Orlando, Florida (1999).

10. M. Abshinova, A. Lopatin, N. Kazantseva, J. Vilcakova and P. Saha, Composites: Part A, 10, 1016 (2007).

11. K.H. Chung and J.H. Lee, Elastom. Compos., 46, 311 (2011). 